EESTI NSV TEADUSTE AKADEEMIA TOIMETISED 1954. III kd., nr. 4 ИЗВЕСТИЯ АКАДЕМИИ НАУК ЭСТОНСКОИ ССР 1954. ТОМ III, № 4

\title{
РАСЧЕТНО-ГРАФИЧЕСКИЙ МЕТОД ОПРЕДЕЛЕНИЯ ВЕЛИЧИНЫ ОБМЕННОИ МОЩНОСТИ МЕЖДУ ЭЛЕКТРОЭНЕРГЕТИЧЕСКИМИ СИСТЕМАМИ
}

\author{
л. А. Алумяэ
}

\section{1. Общие положения}

При проектировании объединений электроэнергетических систем одной из основных задач является определение предполагаемой величины обменной мощности и энергии.

Как известно, обмен мощности и энергии не является постоянным как в годовом, так и в многолетнем разрезе из-за непрерывного изменения 1) гидроэнергетических ресурсов систем, 2) располагаемых мощностей электростанций систем, 3) потребления электроэнергии энергосистемами.

Для того чтобы иметь характеристики обменной мощности и энергии в зависимости от вышеуказанных величин, обычно приходится вести большую вычислительную работу со значительной затратой времени.

Насколько известно автору статьи, в нашей технической литературе до сих пор не поднят вопрос о разработке надежного и простого метода расчета, приемлемого при практическом решении этой задачи. Поэтому целью настоящей работы является разработка практического метода определения обменной мощности и энергии между энергосистемами, причем на первом этапе исследование ограничивается случаем объединения двух систем.

Единственно правильным методом определения обменной мощности между объединяемыми энергосистемами является метод, основанной на принципе экономного распределения нагрузки между всеми работающими электростанциями на основании суммарного графика нагрузки $\left({ }^{1,2}\right)$.

Принцип экономного распределения нагрузки по существу заключается в том, что при любой суммарной нагрузке систем необходимо находить такое распределение нагрузки между всеми электростанциями, работающими в системах, которое в каждый момент времени обеспечит минимальный расход условного топлива по объединению энергосистем.

Для реализации этого принципа на основании расходной характеристики каждой отдельной электростанции $P_{1}=f\left(P_{2}\right)$ составляется функция

$$
\delta=g\left(P_{2}\right),
$$

где $P_{1}$ - подведенная мощность,

$P_{2}$ - полезная мощность,

$\delta=\frac{d P_{1}}{d P_{2}}-$ частичный удельный расход (отношение приращения подведенной мошности к приращенню полезной мощности). 
Функции $f\left(P_{2}\right)$ и $g\left(P_{2}\right)$, как правило, не являются линейными. Однако можно считать, что в пределах определенных интервалов мощностей, экономической зоны и перегрузочной зоны, функция $P_{1}=f\left(P_{2}\right)$ линейна. Очевидно, что в таком случае частичные удельные расходы в пределах этих зон оказываются постоянными * (фиг. 1).

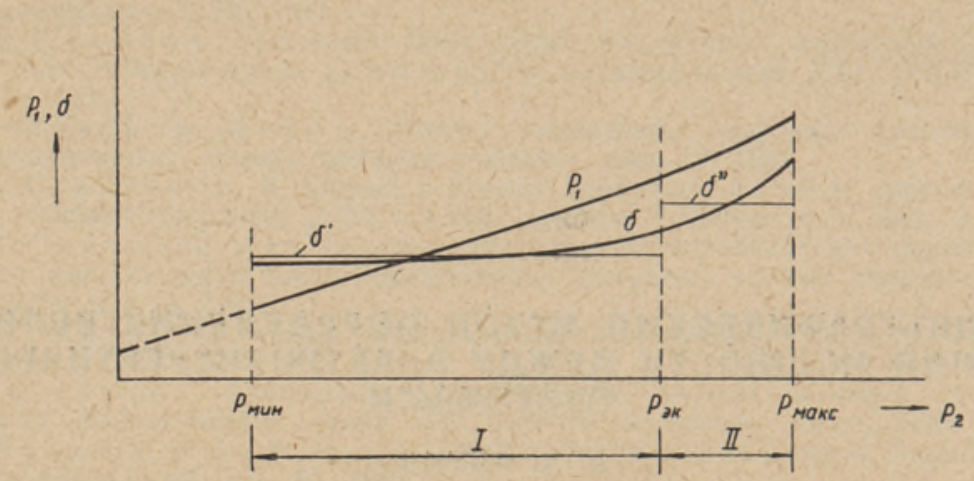

Фиг. 1. Характеристики расхода и частичного удельного расхода электростанции. I - зона экономичных нагрузок, $I I$ - перегрузочная зона, $P_{\text {мнн }}$ - технически-минимальная нагрузка, $P_{\text {эк }}$ экономичная нагрузка, $P_{\text {макс }}$ - максимальная нагрузка, $\delta^{\prime}-$ средний частичный удельный расход в зоне экономичных нагрузок, $\delta^{\prime \prime}$ - то же, в перегрузочной зоне.
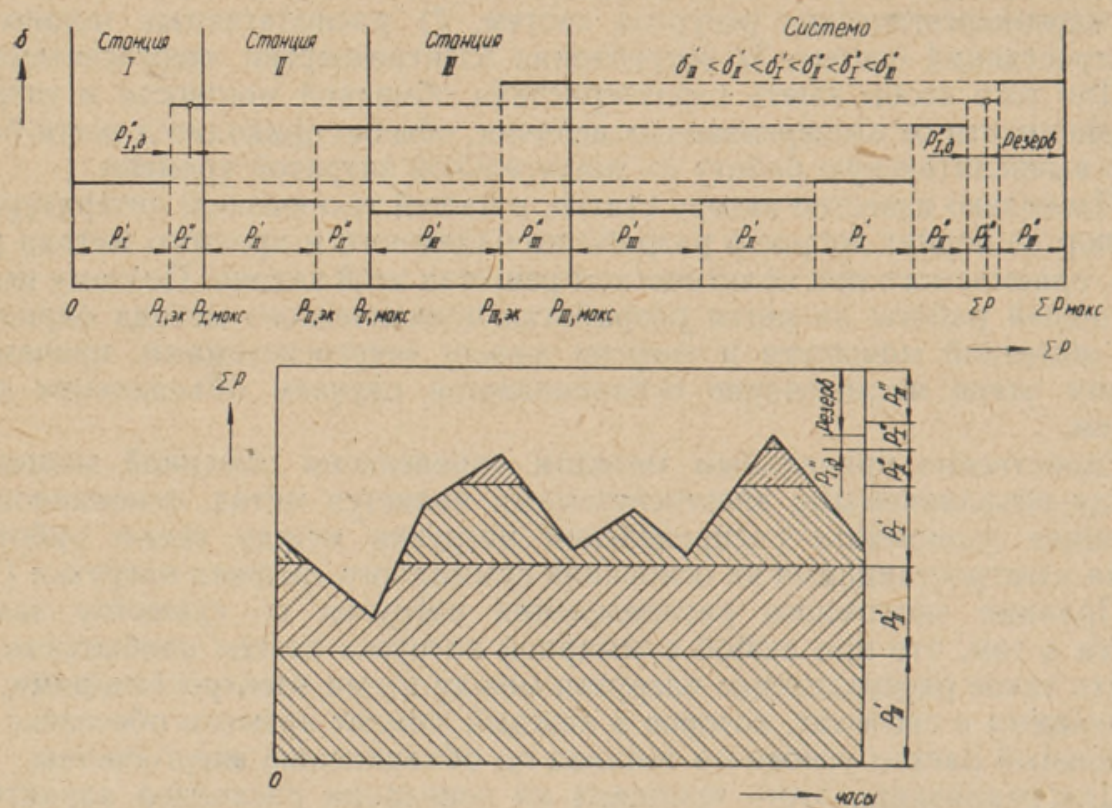

Фиг. 2. Пример распределения нагрузки между тремя параллельно работающими электростанциями по частичным удельным расходам.

Из самого определения частичного удельного расхода следует, что при параллельной работе электростанций и их систем необходимо макси-

* Такое допущение возможно, так как при перспективном проектировании объединений энергосистем отсутствуют точные энергетические характеристики намечаемых к постройке электростанций. 
мально загружать электростанции, имеющие меньший частичный удельный расход.

Следовательно, при изменении суммарной нагрузки систем в небольших пределах изменяется нагрузка только одной электростанции, а другие будут работать с постоянной нагрузкой.

Таким образом, каждая станция покрывает в суммарном графике нагрузки один слой или несколько слоев. Пример с тремя электростанцнями приведен на фиг. 2.

Если в системах работают электростанции, энергетические ресурсы которых для производства электроэнергии лимитированы или заданы, то задача экономичного распределения нагрузки между электростанциями становится намного сложнее.

Задача состоит в том, что эти станции должны: 1) на базе заданных энергетических ресурсов вырабатывать возможно больше электроэнергии, 2) во время пика суммарной нагрузки развивать возможно больше мощности для его покрытия.

Поскольку частичные удельные расходы гидростанций мало зависят от нагрузок и они в любое время готовы для загрузки и разгрузки, то обычно покрытие пика нагрузки и регулирование частоты в энергосистемах возлагается на гидростанции (конечно, могут быть и исключения). Это обстоятельство позволяет теплоэлектростанциям работать длительное время с равномерной нагрузкой и с хорошими экономическими показателями.

Покрытие полупиковой части нагрузки предоставляется, как правило, теплоэлектрическим станциям с худшими экономическими показателями или станциям с заданными расходами топлива.

Ниже рассмотрим роль гидроэлектрических станций в покрытии нагрузки с точки зрения лучшего использования их- установленной мощности.

\section{2. Участие гидроэлектрических станций (ГӘС) в покрытии нагрузки}

Предположим, что ГЭС обладают полным суточным регулированием. Перегрузочную зону агрегатов ГӘС для упрощения не учитываем.

В начале рассмотрим случай, когда в покрытии нагрузки участвует только одна ГЭС.

Возьмем произвольно суточный график нагрузки и построим его интегральную кривую $\left({ }^{3}\right)$ соответственно фиг. 3.

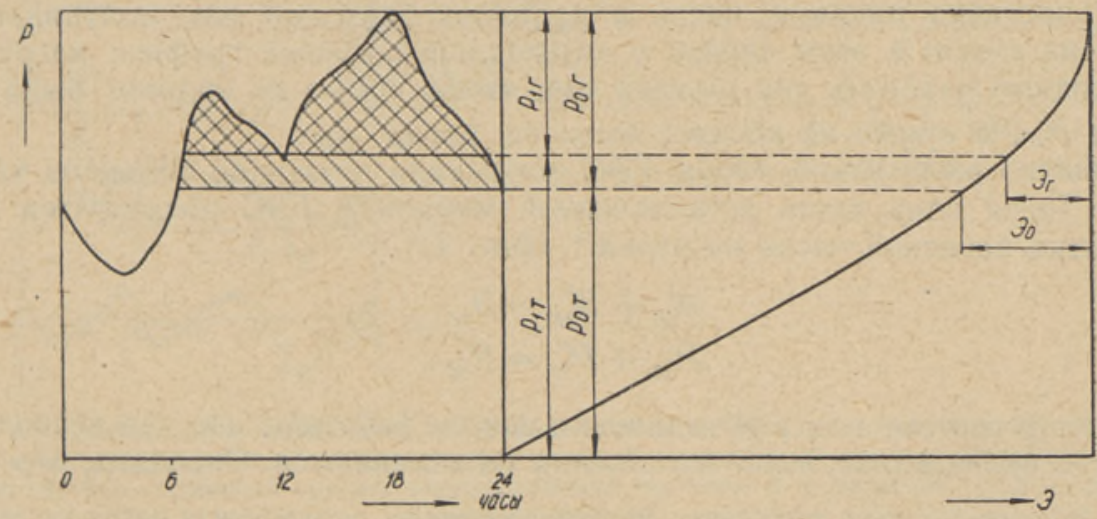

Фнг. 3. Недонспользование установленной мощности ГЭС для покрытия суточного графнка нагрузки при недостатке воды. 
Отделим от пика графика нагрузки отрезок $P_{0}$, соответствующий установленной мощности ГЭС. Этим мы определим и минимальное количество электроэнергии $\exists_{0}$, которое ГЭС должна вырабатывать, чтобы покрывать пик нагрузки своей установленной мощностью $\left(P_{0}\right)$.

В зависимости от действительно возможной выработки электроэнергии $\vartheta_{\Gamma}$, т. е. в зависимости от действительных располагаемых гидроэнергетических ресурсов в сутки, возможны следующие характерные случаи:

1) $\exists_{\Gamma}<\vartheta_{0}$. Это показывает, что во время пика ГЭС неспособна развивать свою установленную мощность. Необходимо увеличить нагрузку теплоэлектрических станций от $P_{0 T}$ до $P_{1 T}$ (фиг. 3 ):

$$
\begin{aligned}
& P_{1 \Gamma}<P_{0 r} \\
& P_{1 T}>P_{0 T} .
\end{aligned}
$$

2) $\vartheta_{\Gamma}=\vartheta_{0}$. Полное использование установленной мощности ГЭС

3) $\exists_{\Gamma}>\boldsymbol{\exists}_{0}$. Полное использование установленной мощности ГЭС, причем она может покрывать:

a) полупиковую часть или базисную и полупиковую части нагрузки; здесь возможно только одно решение - методом подбора необходимо находить у интегральной кривой графика нагрузки такое место, где $\ni_{2 r}$ соответствовало бы $P_{2 \Gamma}=P_{0 г}$ (фиг. 4);

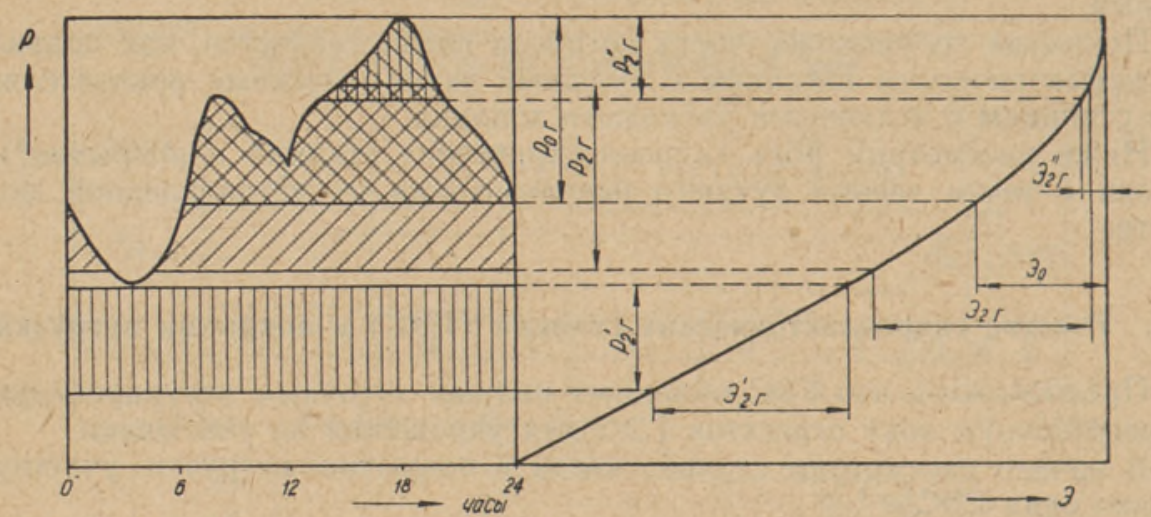

Фиг. 4. Использование установленной мощности ГЭС для покрытия суточного графика нагрузки при избытке воды.

б) частично пиковую часть и частично базисную или полупиковую часть нагрузки; в этом случае у интегральной кривой графика нагрузки необходимо находить два отрезка так, чтобы сумма их ординат была бы равна $P_{0 г}$, а сумма их абсцисс была бы равна $Э_{2 \Gamma}$.

Обычно желательно, чтобы ГЭС покрывала возможно бо́льшую часть пика. Тогда одна часть установленной мощности ГЭС расходуется для покрытия базисной части нагрузки * (фиг. 4):

$$
\begin{aligned}
& \vartheta_{2 \Gamma}^{\prime}+\vartheta_{2 \Gamma}^{\prime \prime}=\vartheta_{2 \Gamma} \\
& P_{2 \Gamma}^{\prime}+P_{2 \Gamma}^{\prime \prime}=P_{0 \Gamma} .
\end{aligned}
$$

Если в системе или в объединении систем работают две или несколько ГЭС, то приведенные выше положения не изменяются. Очевидно, что пик

* Выполнение этого требования могут ограничивать вынужденная нагрузка теплоэлектроцентралей (ТЭЦ), технически-минимальная нагрузка конденсационных электростанций (КЭС) и мощность ГЭС, работающих по водотоку. 
нагрузки покрывается только одной ГЭС, остальные работают в полупиковой или базисной части графика нагрузки. Пик нагрузки будет дан, как правило, на ту ГЭС, которая обладает лучшими условиями для регулирования, т. е. регулирующие бассейны которой бо́льших размеров, колебания горизонтов воды в верхнем и нижнем бьефах при регулировании незначительны, а следовательно, наблюдаются и меньшие колебания напора и меньшие потери выработки энергии. Для лучшего использования установленных мощностей ГЭС могут иметь место и некоторые исключения.

Пользуясь интегральной кривой графика нагрузки, следует методом подбора находить те области работы ГЭС, где их установленная мощность будет использована наиболее эффективно.

То, что сказано о ГЭС, работающих в полупиковой или в базисной части графика нагрузки, полностью действительно и для КЭС с лимитированным или заданным суточным расходом топлива.

\section{3. Методика определения обменной мощности и энергии}

Допустим, что в качестве исходных данных для рассматриваемого нами периода известны спрямленные характеристики электростанций (с учетом собственных нужд), работающих в системах $A$ и $B$, суточные графики нагрузок каждой системы, суммарный суточный график нагрузки объединения систем и выработки электроэнергии в сутки ГЭС (на основании средних эксплуатационных показателей) *.

Теперь можно перейти к решению поставленной задачи с помощью графического метода, изображенного на фиг. 5.

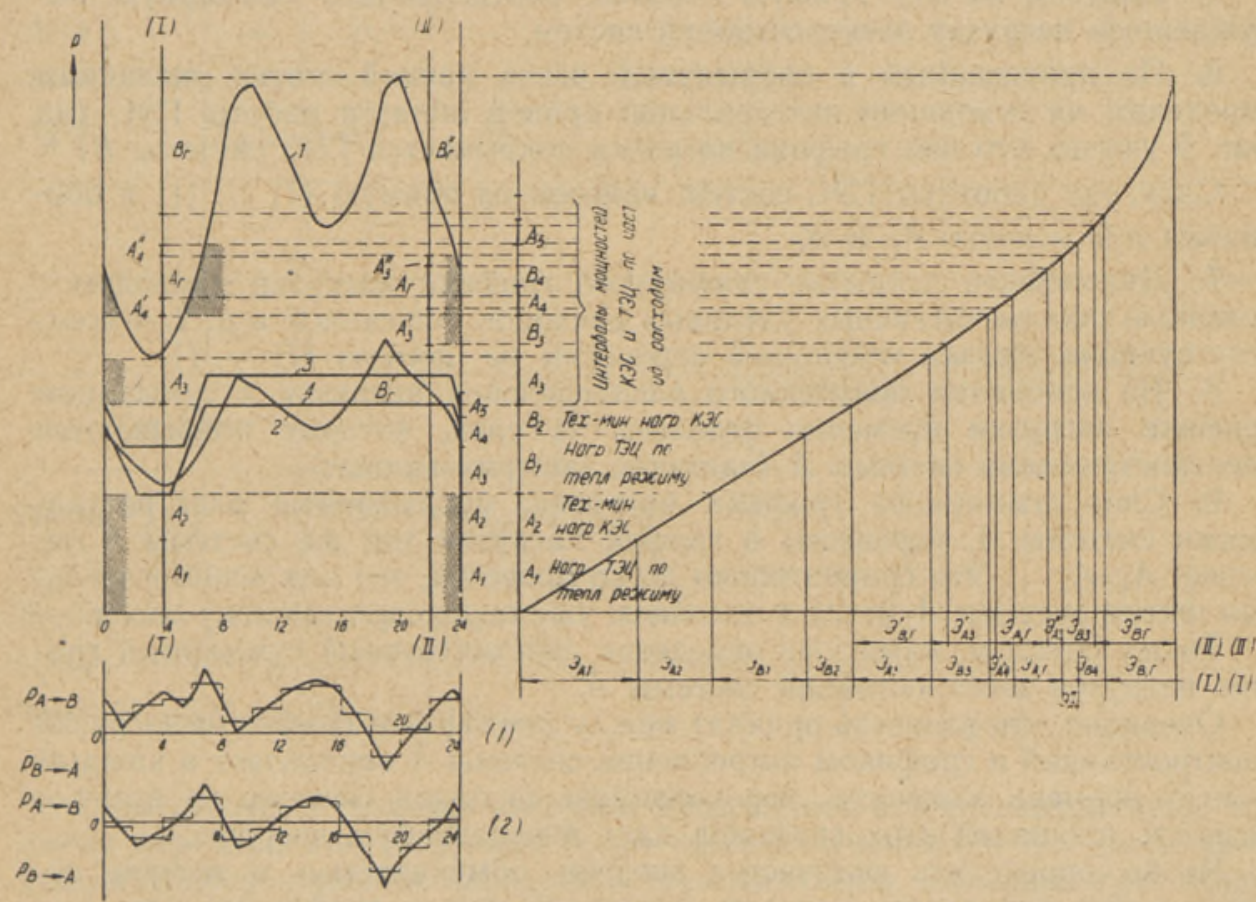

Фиг. 5. Графическое определение обменной мощности между двумя энергосистемами. 1 - суммарный суточный графнк потребления, 2 - суточный график потребления системы $A, 3$ - суточный график электростанций системы $A$ к варианту I, 4 - то же, к варианту II.

* Для упрощения принято, что в каждой системе работает только одна ГЭС. 
Порядок пользования графическим методом:

1. Вычерчиваем суммарный суточный график нагрузки систем, его интегральную кривую и на тех же осях - суточный график нагрузки одной системы (например, системы $A$ ).

2. Построим через нулевую точку интегральной кривой параллельно оси ординат вспомогательную шкалу, на которую наложим:

(I) суммы вынужденных нагрузок электростанций в следующем порядке -

а) ТЭЦ системы $A$ по тепловому режиму $\left(A_{1}\right)$,

б) технически-минимальных КЭС системы $A\left(A_{2}\right)$,

в) ГЭС системы $A$, работающих по водотоку (в наших условиях обычно таких нет),

г) ТЭЦ системы $B$ по тепловому режиму $\left(B_{1}\right)$,

д) технически-минимальных КЭС системы $B\left(B_{2}\right)$,

е) ГЭС системы $B$, работающих по водотоку,

(II) интервалы мощностей КЭС и ТЭЦ обеих систем соответственно порядку увеличения их частичных удельных расходов $\left(A_{3}, B_{3}, A_{4}, B_{4}\right.$ и т. д.).

Отрезки, принадлежащие к системе $A$ ( $A_{1}, A_{2}$ и т. д.), желательно выделить более жирной линией.

3. На правом краю суммарного графика нагрузки построим вторую вспомогательную шкалу, на которую нанесем отрезки мощностей, принадлежащих только к системе $A$, в порядке: $A_{1}, A_{2}$ и т. д.

4. Параллельно оси ординат проведем произвольную прямую (I) ... (I) через суммарный суточный график нагрузки.

5. Нанесем на эту прямую отрезки, определяющие суммарную вынужденную нагрузку электростанций систем.

6. По приведенным в предыдущей части данной статьи указаниям определим на основании интегральной кривой области работы ГӘС (на фиг. 5 учтено, что пик графика нагрузки покрывается ГӘС системы $B$ ) *

Слои, где работают ГӘС систем, нанесем на прямую (I) ... (I) и обозначим для ясности $A_{\Gamma}$ и $B_{\Gamma}$.

7. Дальнейшее покрытие суммарного графика нагрузки проводим с помощью шкалы частичных удельных расходов, описанной в п. 2, и соответствующие отрезки мощностей переносим на прямую (I) . . . (I).

8. На основании полученного распределения нагрузки в суммарном графике нагрузки временно отделяем те слои, которые покрываются электростанциями системы $A$ (частично заштрихованные).

9. Слои суммарного графика нагрузки, покрываемые электростанциями системы $A$, перенесем в график нагрузки той же системы в порядке: $A_{1}, A_{2} \ldots$ Это сравнительно легко провести, так как вспомогательная шкала системы $A$ (п. 3 ) в основном уже определит толщину слоев.

Таким образом, нами уже определен действительный суммарный график нагрузки электростанций системы $A$.

Очевидно, что разность ординат между суммарным графиком нагрузки электростанций и графиком потребления системы $A$ определяет в каждый момент времени мощность, передаваемую из одной системы во вторую; разность площадей этих графиков даст передаваемую энергию.

Чтобы определить количества энергии, обмениваемые в течение суток, следует определить разность площадей между графиками нагрузки

* Большим преимуществом расчетно-графического метода является то, что он позволяет сравнительно быстро определять области работ ГЭС. При прнменении же чисто расчетного метода приходится проделывать огромную работу для того, чтобы путем последовательного приближения получить те же результаты, 
и графиками потребления электростанций. Максимальную передаваемую мощность можно находить прямо из графика методом подбора.

Задача по существу решена и можно переходить к решению следующего варианта, в котором, например, гидроэнергетические ресурсы систем отличаются от таковых в предыдущем варианте. Повторяются все указанные выше операции (в качестве примера на фиг. 5 решен еще второй вариант) *.

При большом числе вариантов сосредоточивание графиков нагрузок электростанций одной системы на один чертеж делает картину распреде ления нагрузок неясной и поэтому целесообразно вычертить кривую обменной мощности для каждого варианта отдельно и на основании этой кривой определить обмен энерғии и максимальную передаваемую мощность (левая нижняя часть фиг. 5).

Обмен энергии целесообразно определить путем средней передаваемой мощности для каждого небольшого промежутка времени, например за 1 час, с нанесением этих значений в таблицу нижеприведенной формы (на фиг. 5 принят промежуток времени в 2 часа). Очевидно, что сумма средних передаваемых мощностей и представляет обмениваемые в сутки количества энергии.

\begin{tabular}{|c|c|c|c|c|c|c|c|c|c|c|c|}
\hline \multirow{4}{*}{ Часы } & \multicolumn{11}{|c|}{ Передаваемая мощность в тыс. квт. } \\
\hline & \multicolumn{4}{|c|}{ Вариант I } & \multicolumn{4}{|c|}{ Вариант II } & \multicolumn{3}{|c|}{ Вариант III } \\
\hline & \multicolumn{2}{|c|}{$\begin{array}{l}\text { Макси- } \\
\text { мальная }\end{array}$} & \multicolumn{2}{|c|}{ Средняя } & \multicolumn{2}{|c|}{$\begin{array}{r}\text { Макси- } \\
\text { мальная }\end{array}$} & \multicolumn{2}{|c|}{ Средняя } & \multirow{2}{*}{$\begin{array}{c}\begin{array}{c}\text { Макси- } \\
\text { мальная }\end{array} \\
A \rightarrow B \mid B \rightarrow A\end{array}$} & \multicolumn{2}{|c|}{ Средняя } \\
\hline & $A \rightarrow B$ & $B \rightarrow A$ & $A \rightarrow B$ & $B \rightarrow A$ & $A \rightarrow B$ & $B \rightarrow A$ & $A \rightarrow B$ & $B \rightarrow A$ & & $A \rightarrow B$ & $B \rightarrow A$ \\
\hline $0-1$ & - & - & $P_{1}$ & - & & & & & & & \\
\hline $1-2$ & - & $P_{B \rightarrow A}$ & - & $P_{2}$ & & & & & & & \\
\hline : & - & - & : & : & & & $f$ & & & & \\
\hline : & - & - & : & : & & & & & & & \\
\hline : & - & - & $\therefore$ & : & & & & & & & \\
\hline $22-23$ & $P_{A \rightarrow B}$ & - & - & $P_{23}$ & & & & & & & \\
\hline $23-24$ & - & - & $P_{24}$ & - & & & & & & & \\
\hline Итого: & $P_{A \rightarrow B}$ & $P_{B \rightarrow A}$ & $\Sigma P_{A \rightarrow B}$ & $\Sigma P_{B \rightarrow A}$ & & & & & & & \\
\hline
\end{tabular}

Если суточные графики нагрузки представить в ступенчатом виде, то линия передаваемой мощности будет также ступенчатой. Но здесь уже нельзя непосредственно определять максимальную передаваемую мощность. Практика применения обоих способов показывает, что отношение абсолютной передаваемой мощности к среднечасовой максимальной

* Целесообразно прямые (I) ... (I), (II) ... (II) и т. д. для каждого варианта сохранить, чтобы облегчить проверку, если это окажется необходимым. 
мощности находится в пределах $1,1-1,3$, причем меньшая величина действительна при большей среднечасовой максимальной мощности. Применение предлагаемой методики с использованием графиков нагрузки в ступенчатом виде является неточным и поэтому ее не следует рекомендовать.

\section{4. Выводы}

Изложенный расчетно-графический метод определения обменной мощности и энергии между электроэнергетическими системами позволяет

1) значительно сокращать время для проведения энергетических расчетов,

2) изображать в наглядной форме обмены мощности и энергии между системами,

3) значительно облегчить расчеты в тех случаях, когда в системах изменяются установленные мощности отдельных электростанций или их энергетические характеристики, так как суммарный суточный график потребления, его интегральная кривая и суточный график системы, взятый за основу, не изменяются.

Применение предложенного метода является особенно эффективным в том случае, когда гидроэнергетические коэффициенты объединяемых систем небольшие (порядка $0,1-0,5$ ), так как базисные и полупиковые части графиков нагрузок остаются в основном неизменными и обмен мощности и энергии в часы низких нагрузок (ночью) для большинства вариантов остается одинаковым, за исключением многоводных периодов (вариант II на фиг. 5).

\section{Ннститут энергетики Академии наук Әстонской ССР \\ Поступила в редакцию 28 VIII 1954}

\section{ЛИТЕРАТУРА}

1. В. В. Болотов, Теоретические основы выбора экономического режима сложной электроэнергетической системы, Изд. АН СССР, 1947.

2. В. М. Г о р н ш те й н, Наивыгоднейшее распределение нагрузок между параллельно работающими электростанциями, Госэнергоиздат, М.-Л., 1949.

3. А. А. Морозо в, Использование водной энергии, Госэнергоиздат, М.-Л., 1948. 